\title{
Validation of self-reported folic acid use in a multiethnic population: results of the Amsterdam Born Children and their Development study
}

\author{
Jonne J Sikkens ${ }^{1}$, Manon van Eijsden ${ }^{2,3}$, Gouke J Bonsel ${ }^{4}$ and Martina C Cornel ${ }^{1, *}$ \\ 'Department of Clinical Genetics, EMGO Institute for Health and Care Research, VU University Medical \\ Center, BS7, D423, PO Box 7057, 1007 MB Amsterdam, The Netherlands: '2Department of Epidemiology, \\ Documentation and Health Promotion, Public Health Service of Amsterdam, The Netherlands: ${ }^{3}$ Department of \\ Social Medicine, Academic Medical Centre, University of Amsterdam, The Netherlands: ${ }^{4}$ Department of Public \\ Health, Institute of Health Policy and Management, Erasmus University, Rotterdam, The Netherlands
}

Submitted 4 May 2010: Accepted 12 January 2011: First published online 16 February 2011

\begin{abstract}
Objective: To assess folic acid supplementation rates and validate the self-reporting of folic acid supplement use among pregnant women in a multiethnic cohort. Design: Secondary analysis of a prospective cohort study. Setting: Self-reported folic acid supplement use in the Amsterdam Born Children and their Development study cohort was compared with serum folate concentrations using non-parametric trend analysis and linear and logistic regression. Subjects: A total of 4234 pregnant women of various ethnic backgrounds. Results: Serum folate levels showed a significant positive linear trend as reported use of folic acid increased $(P<0 \cdot 001)$, which was supported by linear regression $(r=0 \cdot 49)$. Odds of having low serum folate concentration decreased with reported early start of folic acid intake. Young, multiparous or non-Western women reported less pre-conception folic acid intake. Non-Western women showed lower serum folate concentrations. The overall rate of over-reporting, i.e. serum folate concentrations $\leq 20 \mathrm{nmol} / \mathrm{l}$ while reporting the use of folic acid supplements, was $20.7 \%$. Women of Surinamese and Moroccan ancestry had higher odds of overreporting $(\mathrm{OR}=2 \cdot 3 ; 95 \% \mathrm{CI} 1 \cdot 5,3 \cdot 5$ and $\mathrm{OR}=2 \cdot 3 ; 95 \% \mathrm{CI} 1 \cdot 3,4 \cdot 0$, respectively). The odds for Surinamese women remained significant after adjusting for the onset of supplement use, parity and age (OR $=1 \cdot 7 ; 95 \%$ CI $1 \cdot 1,2 \cdot 6)$.

Conclusions: Although self-reporting is a valid method for assessing folic acid supplement use in a multiethnic population, some participants do over-report. Surinamese and possibly Moroccan women appear to over-report more often. Rates of supplementation are low, especially in non-Western women. This suggests the need for intensifying current campaigns or perhaps even additional advice to start or continue to use folic acid post-conceptionally.
\end{abstract}

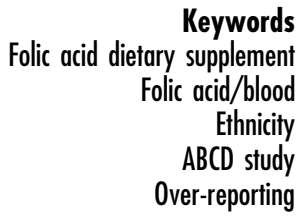

A low maternal serum folate concentration before conception and during the first weeks after conception is associated with an increased risk of fetal neural tube defects (NTD) and other non-NTD birth defects ${ }^{(1-3)}$ and can be prevented by the intake of folic acid ${ }^{(4)}$. Adequate folic acid supplementation has been shown to substantially increase folate serum levels ${ }^{(5)}$. Although prophylactic use is widely recommended in public campaigns, recent studies suggest little or no improvement in NTD prevalence by self-intake ${ }^{(6)}$, which raises the question as to whether behavioural change did occur.

Ideally, adherence to the recommendations is measured by serum folate status in conjunction with reported use; however, most epidemiological studies rely on self- report only, as this is less invasive and cheap. Being a socially desirable behaviour, self-reported use is prone to response bias, which raises questions regarding the actual validity of this measure. A study by Burton et al. ${ }^{(7)}$ showed that, although intake reports and folate levels are well correlated, over-reporting is present in $7 \%$ of respondents. However, Burton et al.'s ${ }^{(7)}$ study was limited to mainly white participants. Although evidence suggests that race or ethnicity does not influence socially desirable responding by itself, factors such as divorce rate, birth rate, level of education and rate of employment do influence socially desirable responding, and these factors are often not similar across ethnic groups ${ }^{(8-10)}$. Therefore, ethnic differences in over-reporting of folic acid 
supplementation could be expected. Insight into these mechanisms is essential in order to improve public health policies. Using data from a multiethnic cohort in the Netherlands - the Amsterdam Born Children and their Development (ABCD) cohort - we investigated the rates of supplementation and the validity of self-reported folic acid supplementation in terms of over-reporting.

\section{Methods}

\section{Data collection}

Data were obtained from the ABCD study in 2003 and 2004. The present cohort study included pregnant women at their first antenatal control visit to a health professional. Consenting women had a questionnaire sent to their homes a few weeks later. In addition, women were asked to participate in a biomarker study. This entailed a blood sample being collected in conjunction with routine prenatal screening following the control visit. The blood sample collection procedure included samples sent by mail or courier, which could lead to a maximum delay of $28 \mathrm{~h}$ between collection and processing. However, this diminished precision of estimates did not jeopardise the integrity of epidemiological research ${ }^{(11)}$. Serum folate concentrations were measured by immunoassay with chemiluminescence detection on the Advia Centaur System (Bayer Group, Leverkusen, Germany) ${ }^{(12)}$. Values of serum folate above the detection limit of $63 \mathrm{nmol} / 1$ were set to $64 \mathrm{nmol} / \mathrm{l}$.

\section{Measures}

Responses to the question 'What is your country of birth?' were used to classify women into groups according to their ethnic background. Responses to the question 'Which community do you consider yourself to belong to?' were available to further classify respondents into ethnic groups similar to the study by Burton et al. ${ }^{(7)}$. The questionnaire contained the following three questions assessing folic acid supplement use (possible answers in brackets): (i) 'Did you use folic acid supplements or folic acid containing multivitamins before or during your pregnancy?' (yes; no), if yes: (ii) 'When did you start taking folic acid?' (before the pregnancy; as soon as I knew I was pregnant; later during the pregnancy) and (iii) 'When did you stop taking folic acid?' (before the pregnancy; as soon as I knew I was pregnant; later during the pregnancy; I have not stopped yet). The responses to these questions were combined into four groups according to the onset of folic acid supplement use (Table 1).

\section{Analysis}

The Wilcoxon-type test for trend was used to test for a linear trend in serum folate across groups ${ }^{(13)}$. Linear regression was used to compare serum folate concentrations between folic acid supplement use groups, adjusting for parity, age and ethnicity. In addition, a dichotomous
}

variable was created to indicate low serum folate status. For this we used a cut-off limit of $20 \mathrm{nmol} / 1$ because 2 months of adequate folic acid supplementation has been shown to lead to a minimum serum folate level of $20 \mathrm{nmol} / \mathrm{l}^{(5)}$. Logistic regression was used to compare the odds of low serum folate status between folic acid supplement use groups, adjusting for parity, age and ethnicity.

To assess the effects of ethnicity, we first compared serum folate concentrations and the occurrence of low serum folate across ethnic groups. Using Levene's statistic, we assessed that variances of serum folate concentrations were not homogeneous across groups. Therefore, we compared serum folate concentrations over ethnic groups by testing for equality of means by Brown-Forsythe and we compared groups using the Tamhane test. A logistic regression model (adjusting for folic acid supplement use, age and parity) was used to compare odds of having low serum folate level $(\leq 20 \mathrm{nmol} / \mathrm{l})$.

To assess over-reporting, a variable was constructed by allocating women to one of two groups: (i) women reporting folic acid use while their serum folate concentration showed a value $\leq 20 \mathrm{nmol} / 1$ and (ii) all other women. Finally, to assess over-reporting in ethnic groups, a logistic regression model was used to compare the odds of having a low serum folate level $\leq 20 \mathrm{nmol} / 1$ in women who reported folic acid supplement use, adjusting for onset of folic acid supplement use, age and parity. Consequently, women not reporting folic acid use are missing from this specific analysis. OR was reported as the measure of association. All analyses were performed using the Statistical Package for the Social Sciences statistical software package for Windows version $15 \cdot 0 \cdot 1$ (SPSS Inc., Chicago, IL, USA).

\section{Results}

\section{Subjects}

Of 12373 pregnant women, 8266 (67\%) returned a completed questionnaire, and of these women 4389 (53\%) also provided a blood sample. Cases with incomplete information on folic acid supplement use or with an inconclusive blood sample were excluded, leaving 4234 cases for analysis. The median age was 32 (range: $15-44$ ) years and 2452 (58\%) women were nulliparous. Median gestational age was 13 weeks at the time of blood collection and 16 weeks at questionnaire completion. Most women were born in the Netherlands (69\%), followed by Morocco (5\%), Suriname (5\%), Turkey (3\%), Ghana (1\%) and the Dutch Antilles and Aruba (1\%), with $9 \%$ and $7 \%$ being born in another Western or non-Western country, respectively. Using the question "Which community do you consider yourself to belong to?' $69 \%$ of women classified themselves as belonging to the indigenous, white population.

\section{Reported folic acid supplement use}

Only $39.9 \%$ of women used folic acid as recommended: before conception or at least before they knew they were 
pregnant (Table 1). In all, $43 \%$ of nulliparous or primiparous women reported using folic acid as recommended, compared with $20 \%$ of multiparous women. Women aged $\geq 25$ years used folic acid as recommended in $45 \%$ of cases, whereas this was the case in only $11 \%$ of women aged $<25$ years. Rates of folic acid use as recommended were much lower in non-indigenous women compared with women born in the Netherlands, with rates among women of nonWestern ancestry not exceeding 25\% (Table 2).

\section{Validity of self-reported folic acid supplement use}

There was a significant linear trend in serum folate levels as reported duration of use of folic acid supplements increased $(Z=36 \cdot 5, \quad P<0 \cdot 001)$. Use of folic acid supplements was positively correlated with serum folate concentration $(r=0 \cdot 49, P<0 \cdot 001)$. After adjusting for parity, age and ethnicity, the positive correlation remained significant $(r=0 \cdot 51$, $P<0 \cdot 001)$. Table 1 shows that folic acid supplement use before pregnancy, after being aware of pregnancy and later in pregnancy was associated with a lower prevalence of serum folate $\leq 20 \mathrm{nmol} / \mathrm{l}(P<0 \cdot 001)$, which remained significant after adjusting for parity, age and ethnicity $(P<0.001)$. Over-reporting was present in $20.7 \%$ of cases.

\section{Serum folate in etbnic groups}

Serum folate concentrations varied significantly according to maternal country of birth (Brown-Forsythe ( $\mathrm{df}=7928$ ), $P<0 \cdot 001$; for between-group comparisons see Table 2 ). Women of non-indigenous ancestry had significantly more frequent serum folate concentrations $\leq 20 \mathrm{nmol} / \mathrm{l}$, except for women of Western ancestry. This remained significant after adjusting for parity and age (for OR, see Table 2). When adjusting for onset/start of supplement use, parity and age, women of Surinamese and Moroccan ancestry had significantly more frequent serum folate concentrations $\leq 20 \mathrm{nmol} / \mathrm{l}(\mathrm{OR}=2 \cdot 0 ; 95 \% \mathrm{CI} 1 \cdot 3,2 \cdot 8$ and $\mathrm{OR}=1 \cdot 7 ; 95 \% \mathrm{CI} 1 \cdot 1,2 \cdot 6$, respectively).

\section{Over-reporting in etbnic groups}

Among women reporting folic acid supplement use, women of either Surinamese or Moroccan ancestry had serum folate level $\leq 20 \mathrm{nmol} / 1$ significantly more often, interpreted as over-reporting ( $\mathrm{OR}=2 \cdot 3 ; 95 \%$ CI $1 \cdot 5,3 \cdot 5$ and $\mathrm{OR}=2 \cdot 3 ; \quad 95 \%$ CI $1 \cdot 3,4 \cdot 0$, respectively). After adjusting for onset of supplement use, parity and age, this remained significant only for women of Surinamese ancestry $(\mathrm{OR}=1 \cdot 7 ; 95 \% \mathrm{CI} 1 \cdot 1,2 \cdot 6)$. Complete results are shown in Table 3.

\section{Discussion}

\section{Strengths and limitations}

Folic acid supplementation use before conception is recommended because it provides health benefits for the unborn child. Questions have arisen regarding whether 
women are complying with this recommendation. Previous research in which folic acid supplement use was evaluated has generally used self-report measures for quantification. However, until now these measures have been validated in only one previous study. That study used non-parametric trend analysis and was conducted in a mainly white population $^{(7)}$. Self-report measures leave room for overreporting. The present study aimed at validating a self-report questionnaire in a large multiethnic cohort using non-parametric trend analysis as well as linear and logistic regression. In the current cohort, $69 \%$ of women classified themselves as belonging to the indigenous, white population compared with $80 \%$ in the study by Burton et $\mathrm{al}^{(7)}$. Response rates in the present study were higher than the response rates in comparable multiethnic surveys ${ }^{(14,15)}$. Nevertheless, the possibility of selection bias exists. Because the blood sample procedure allowed for degradation of folate concentrations before analysis, exact levels could not be ascertained; however, as reported by van Eijsden et $a l^{(11)}$, it still allowed for correct classification. Contrary to our study, the study from which our cut-off limit of $\leq 20 \mathrm{nmol} / \mathrm{l}$ originated was based on subjects with unexplained pregnancy loss who might have lower levels of serum folate than other women ${ }^{(5)}$. This may have possibly caused the cut-off limit to be lower than it should have. Consequently, our over-reporting outcomes could be an underestimation. On the other hand, this would have led to fewer women being falsely labelled as over-reporting.

\section{Reported folic acid supplement use}

Only $39.9 \%$ of women reported the use of folic acid supplements as recommended: before conception or at least before they knew they were pregnant. Rates were lower for women aged $<25$ years and for multiparous women, as described in the literature ${ }^{(16)}$. Furthermore, non-Western women also showed low rates of reported folic acid supplement use as recommended, which has been reported previously ${ }^{(17,18)}$.

\section{Validity of self-reported folic acid supplement use}

Both the linear relationship and the moderately strong positive association between serum folate levels and reported use of folic acid supplements suggest that as women take folic acid, their serum folate levels increase correspondingly. Combined with the lower probability of a serum folate concentration $\leq 20 \mathrm{nmol} / 1$ if women report supplement use, this suggests that the self-reported use of folic acid supplements is a valid measure of folate status. The latter effect is stronger with early start of use, which is a very important point because the majority of research focuses on the possible effects of folate deficiency and less so on the spectrum of serum folate concentrations.

Women reportedly using folic acid as recommended had an OR of $0 \cdot 07(95 \%$ CI $0 \cdot 05,0 \cdot 08)$ for low serum folate concentration $(\leq 20 \mathrm{nmol} / \mathrm{l})$. However, serum folate concentration $\leq 20 \mathrm{nmol} / 1$ was found in $19 \cdot 4 \%$ of these 
Table 3 Odds of over-reporting on folic acid supplementation (serum folate concentration $\leq 20 \mathrm{nmol} / \mathrm{l}$ ) in women who reported folic acid supplement uset according to maternal country of birth, Amsterdam Born Children and their Development study

\begin{tabular}{lrrccr}
\hline Country of birth & $n$ & OR & $95 \% \mathrm{Cl}$ & Adjusted OR & $95 \% \mathrm{Cl}$ \\
\hline The Netherlands & 2529 & $1 \cdot 0$ & & $1 \cdot 0$ & $1 \cdot 7$ \\
Suriname & 93 & $2 \cdot 3$ & $1 \cdot 5,3 \cdot 5$ & $1 \cdot 6$ \\
Dutch Antilles and Aruba & 29 & $1 \cdot 2$ & $0 \cdot 6,2 \cdot 7$ & $1 \cdot 0$ & $0 \cdot 4,2 \cdot 3$ \\
Turkey & 33 & $1 \cdot 6$ & $0 \cdot 8,3 \cdot 2$ & $0 \cdot 8$ & $0 \cdot 4,1 \cdot 8$ \\
Morocco & 53 & $2 \cdot 3$ & $1 \cdot 3,4 \cdot 0$ & $1 \cdot 5$ & $0 \cdot 8,2 \cdot 6$ \\
Ghana & 8 & $1 \cdot 7$ & $0 \cdot 4,7 \cdot 0$ & $1 \cdot 0$ & $0 \cdot 2,4 \cdot 7$ \\
Other non-Western & 135 & $1 \cdot 3$ & $0 \cdot 9,1 \cdot 9$ & $1 \cdot 0$ & $0 \cdot 6,1 \cdot 4$ \\
Other Western & 301 & $0 \cdot 9$ & $0 \cdot 7,1 \cdot 2$ & $0 \cdot 9$ & $0 \cdot 7,1 \cdot 2$ \\
\hline
\end{tabular}

tWomen not reporting folic acid supplement use are missing in this table.

$\ddagger$ Corrected for onset of folic acid supplement use, parity and age.

women. Women who reported taking supplements only later during pregnancy (which could be for a very short period of time) presented with low serum folate level in $49 \cdot 6 \%$ of cases. These high proportions could perhaps be explained by the time gap between the moment of blood collection (median $=13$ weeks) and questionnaire completion (median $=16$ weeks), during which period women could have started and consequently reported using supplements. Over-reporting (present in $20.7 \%$ of cases) could also add to this effect. However, over-reporting was similar to the report by Burton et al. ${ }^{(7)}$ when comparing over-reporting rates using the cut-off limit used by that study $(14 \mathrm{nmol} / 1): 8 \cdot 9 \%$ v. $7 \%$.

\section{Serum folate in etbnic groups}

Serum folate concentrations were significantly lower in all non-indigenous groups compared with those in indigenous women (except for women born in the Dutch Antilles and Aruba). In addition, non-Western women also had higher odds of having low serum folate concentration. We will discuss the following possible causes: folate consumption, folate degradation due to time gap, genetic differences and language skills. These low concentrations are not surprising considering the low supplementation rates among these women. However, when adjusting for supplementation, women of Surinamese and Moroccan ancestry still had higher odds of having a low serum folate concentration, even when adjusting for parity and age. A possible factor responsible for these findings is a diet low in folate. Women having either Moroccan or Turkish ancestry are reported to have a diet low in vegetables (which generally contain folate). However, the opposite is the case for women of Surinamese ancestry $^{(19,20)}$. Another factor could be the degradation of folic acid during the time gap between collection and processing. The length of this gap was determined by the location of the practice of the health professional where the blood was collected. Non-indigenous women consulted health professionals in hospitals more often, who processed the blood samples faster. However, in post hoc analysis no significant difference between hospitaland non-hospital-processed serum folate was found
$(P=0 \cdot 95)$. Recent research has shown that genetic polymorphisms such as the MTHFR $677 \mathrm{C} \rightarrow \mathrm{T}$ genotype are associated with low serum folate ${ }^{(21)}$. Prevalence of these polymorphisms varies between ethnic groups but specific data on the non-indigenous groups in the Netherlands are not yet available. Finally, another factor could be overreporting due to other reasons, which are discussed below.

\section{Over-reporting in etbnic groups}

Women having Surinamese or Moroccan ancestry overreported significantly more frequently $(\mathrm{OR}=2 \cdot 3 ; 95 \% \mathrm{CI}$ $1 \cdot 5,3.5$ and $\mathrm{OR}=2 \cdot 3 ; 95 \% \mathrm{CI} 1 \cdot 3,4 \cdot 0$, respectively), but the adjusted OR was significant only for women of Surinamese ancestry $(\mathrm{OR}=1 \cdot 7 ; 95 \% \mathrm{CI} 1 \cdot 1,2 \cdot 6)$. Because the present analysis was performed in a smaller population (women reporting no use were excluded), it is possible that a small sample size caused the loss of significance for the already small group of women of Moroccan ancestry. A seemingly logical explanation for the higher odds of overreporting by non-indigenous women would be insufficient language skills, which could have led to misinterpretation of the questionnaire. However, this is deemed very unlikely because questionnaires were available in their own language and translators were available for oral administration. In addition, women of Surinamese ancestry generally have good skills in Dutch, especially when compared with women of other non-Western ancestry. Nevertheless, the possible answers to the question 'When did you start taking folic acid?' in the questionnaire were not quantitatively exact and may have left room for error. A search of the literature yielded one article focusing on the validity of self-reported pneumococcal vaccination in the USA. That study found effects on the validity of reporting related to ethnicity, but differences in over-reporting between whites, blacks and Latinos were not significant ${ }^{(22)}$. This calls for more research to assess self-reporting by women of Surinamese ancestry.

\section{Implications for bealtb policy}

The above findings suggest that self-reporting is a valid method of assessing folic acid supplementation in a multiethnic population. However, it should be taken into account that among women reporting supplement use as 
recommended, low serum folate levels are still present in $20 \%$ of cases. However, over-reporting in the present study was similar to a previous result in the literature. The finding that Surinamese women tend to over-report more cannot yet be explained and requires further study, particularly into the role of dietary and genetic factors. The low rates of women taking folic acid supplements as recommended show that current public health campaigns are failing to reach their goals, especially among nonWestern women, young women and multiparous women. These results call for improving or modifying current efforts. This may include advising women to take supplements after conception as well, as women who start taking supplements after knowing that they are pregnant were found to have relatively high concentrations of folic acid. Although this may not be beneficial for the prevention of NTD because the neural tube closes at approximately 4 weeks after conception, it may still have a positive influence on other unfavourable pregnancy outcomes such as placenta-mediated diseases (preeclampsia, placental abruption, spontaneous abortion), low birth weight or premature birth ${ }^{(23-26)}$. This suggests that pregnant women should be advised to start taking folic acid supplements or continue to use it, after conception. In addition, this may help to prevent adverse events in subsequent pregnancies, as past research has shown adverse outcomes in closely spaced pregnancies to be associated with folate deficiency ${ }^{(27)}$.

\section{Acknowledgements}

The study was approved by the medical ethics committee of the VU University Medical Center, Amsterdam, the Netherlands. The Amsterdam Born Children and their Development (ABCD) study is funded by the Netherlands Organisation for Health Research and Development (ZonMw). The authors have no conflict of interest to declare. J.J.S. is credited with creating the study design and with data processing, planning and performing all statistical analyses except parts of the regression analysis, as well as with processing and interpreting results and writing the article. M.v.E. contributed towards collecting the ABCD study data, designing the study, data processing, planning and performing parts of the regression analyses, and was also actively involved in interpreting the results and advising on and co-writing the article. G.J.B. advised on statistical analysis and design, interpreted the results and advised on and co-wrote parts of the article. M.C.C. provided valuable advice on statistical analysis and design, and is also credited with interpreting the results, co-writing the article and advising on and supervising the research project. The authors thank M.F. van der Wal for his contributions to previous versions of the present article, H.J. Blom for his useful comments and P.D. Bezemer for his valuable statistical advice.

\section{References}

1. Wilcox AJ, Lie RT, Solvoll K et al. (2007) Folic acid supplements and risk of facial clefts: national population based case-control study. BMJ 334, 433-434.

2. Tamura $\mathrm{T} \&$ Picciano MF (2006) Folate and human reproduction. Am J Clin Nutr 83, 993-1016.

3. Canfield MA, Collins JS, Botto LD et al. (2005) Changes in the birth prevalence of selected birth defects after grain fortification with folic acid in the United States: findings from a multi-state population-based study. Birth Defects Res A Clin Mol Teratol 73, 679-689.

4. MRC Vitamin Study Research Group (1991) Prevention of neural tube defects: results of the Medical Research Council Vitamin Study. Lancet 338, 131-137.

5. Nelen WLDM, Blom HJ, Thomas CMG et al. (1998) Methylenetetrahydrofolate reductase polymorphism affects the change in homocysteine and folate concentrations resulting from low dose folic acid supplementation in women with unexplained recurrent miscarriages. J Nutr 128, 1336-1341.

6. Busby A, Abramsky L, Dolk H et al. (2005) Preventing neural tube defects in Europe: a missed opportunity. Reprod Toxicol 20, 393-402.

7. Burton A, Wilson S \& Gillies AJ (2001) Folic acid: is self reported use of supplements accurate? J Epidemiol Community Health 55, 841-842.

8. Edwards DW (1974) Blacks versus Whites: when is race a relevant variable? J Pers Soc Psychol 29, 39-49.

9. Hébert JR, Peterson KE, Hurley TG et al. (2001) The effect of social desirability trait on self reported dietary measures among multi-ethnic female health center employees. Ann Epidemiol 11, 417-427.

10. Twenge JM \& Im C (2007) Changes in the need for social approval, 1958-2001. J Res Pers 41, 171-189.

11. van Eijsden M, van der Wal MF, Hornstra G et al. (2005) Can whole-blood samples be stored over 24 hours without compromising stability of C-reactive protein, retinol, ferritin, folic acid, and fatty acids in epidemiologic research? Clin Chem 51, 230-232.

12. Owen WE \& Roberts WL (2003) Comparison of five automated serum and whole blood folate assays. Am J Clin Pathol 120, 121-126.

13. Cuzick J (1985) A Wilcoxon-type test for trend. Stat Med $\mathbf{4}$, 87-90.

14. Galea S \& Tracy M (2007) Participation rates in epidemiologic studies. Ann Epidemiol 17, 643-653.

15. Jaddoe VWV, Mackenbach JP, Moll HA et al. (2006) The Generation R Study: design and cohort profile. Eur J Epidemiol 21, 475-484.

16. de Walle HE \& de Jong-van den Berg LT (2008) Ten years after the Dutch public health campaign on folic acid: the continuing challenge. Eur J Clin Pharmacol 64, 539-543.

17. Bakker MK, Cornel MC \& de Walle HE (2003) Awareness and periconceptional use of folic acid among non-western and western women in the Netherlands following the 1995 publicity campaign. Ned Tijdschr Geneeskd 147, 2426-2430.

18. van Eijsden M, van der Wal MF \& Bonsel GJ (2006) Folic acid knowledge and use in a multi-ethnic pregnancy cohort: the role of language proficiency. BJOG 113, 1446-1451.

19. Palsma AH, Nicolaou M, van Dam RM et al. (2006) De Voeding van Turkse en Marokkaanse Nederlandse in de leeftijd van 18-30 jaar: prioriteiten voor voedingsinterventies. TSG 84, 415-422.

20. Nicolaou M, van Dam RM \& Stronks K (2006) Acculturation and education level in relation to quality of the diet: a study of Surinamese South Asian and Afro-Caribbean residents of the Netherlands. J Hum Nutr Diet 19, 383-393. 
21. Yang QH, Botto LD, Gallagher M et al. (2008) Prevalence and effects of gene-gene and gene-nutrient interactions on serum folate and serum total homocysteine concentrations in the United States: findings from the third National Health and Nutrition Examination Survey DNA Bank. Am J Clin Nutr 88, 232-246.

22. Gordon NP, Wortley PM, Singleton JA et al. (2008) Race/ ethnicity and validity of self reported pneumococcal vaccination. BMC Public Health 8, 227.

23. Ray JG \& Laskin CA (1999) Folic acid and homocyst(e)ine metabolic defects and the risk of placental abruption, preeclampsia and spontaneous pregnancy loss: a systematic review. Placenta 20, 519-529.
24. Wen SW, Chen XK, Rodger M et al. (2008) Folic acid supplementation in early second trimester and the risk of preeclampsia. Am J Obstet Gynecol 198, 45.e1-45.e7.

25. Sram RJ, Binkova B, Lnenickova Z et al. (2005) The impact of plasma folate levels of mothers and newborns on intrauterine growth retardation and birth weight. Mutat Res 591, 302-310.

26. Siega-Riz AM, Savitz DA, Zeisel SH et al. (2004) Second trimester folate status and preterm birth. Am J Obstet Gynecol 191, 1851-1857.

27. van Eijsden M, Smits LJM, van der Wal MF et al. (2008) Association between short interpregnancy intervals and term birth weight: the role of folate depletion. Am J Clin Nutr 88, 147-153. 